\title{
Improving the Multiple Access Method of CSMA/CA Home Networks
}

\author{
Miguel Elias M. Campista, Luís Henrique M. K. Costa, and Otto Carlos M. B. Duarte* \\ Universidade Federal do Rio de Janeiro - PEE-COPPE/DEL-POLI \\ Grupo de Teleinformática e Automação - Rio de Janeiro - RJ - Brasil \\ E-mails: \{miguel,luish,otto\}@gta.ufrj.br
}

\begin{abstract}
A home network is a communication system that aims to interconnect household appliances and share the access to the Internet. This work proposes a novel mechanism which is able to improve the multiple access method of home networks. The Contention window Proactive Increase (CPI) mechanism avoids collisions by increasing the number of times the backoff procedure is called. We applied the CPI mechanism to the IEEE 802.11 and HomePlug standards given their similar access methods. We show the efficiency of the proposed mechanism evaluating through simulations the network throughput gains compared to the original standards.
\end{abstract}

\section{Introduction}

A home network is a LAN that interconnects household appliances and provide Internet access at high rates. Due to upcoming applications such as interactive games, voice and video transmissions, a home network must also guarantee quality of service and available bandwidth. There are different contending home-network technologies. Among them, the "no-new-wires" solutions, such as HomePlug [7] and HomePNA [1], have the low cost as major advantage. Another low-cost alternative is the wireless LAN, whose main standard is IEEE 802.11 [4].

Cabling may be decisive when choosing the home network to be installed because it represents a large part of the final installation cost. HomePlug and HomePNA avoid expenses using, respectively, the existent electrical and telephone wiring infrastructure. IEEE 802.11 does not need a cabling infrastructure because it uses RF propagation.

The medium characteristics of home networks are different. The phone line uses twisted pair wiring, which provides lower attenuation and interferences than the electrical wiring and the wireless channel. Nevertheless, HomePNA connectivity is limited to the number of phone outlets in a residence, which is usually smaller than the number

* This work has been supported by FAPERJ, CNPq, CAPES, FINEP, FUNTTEL, and RNP. of electrical outlets. Thus, home networks using PowerLine Communications (PLC) and IEEE 802.11 technologies are very suitable for domestic environments. On the other hand, transmissions over the electrical wiring and RF propagation suffer with attenuation and interferences, which limit the bandwidth.

Mechanisms which increase the performance of IEEE 802.11 and HomePlug are of utmost importance. These mechanisms must overcome the constraints of the wireless and electrical channels. To the best of our knowledge, works aiming to improve HomePlug are not found in the literature. Nevertheless, proposals that enhance IEEE 802.11 have been made. Tay and Chua [8] developed a mathematical model which allows the evaluation of the best $C W_{\min }$ and $C W_{\max }$. Kuo and Kuo [5] proposed a simple backoff scheme to distributed coordination function and an analytical model to analyze the throughput and delay performance of the new scheme. Bononi et al. [2] proposed a distributed contention-control mechanism to avoid collision and improve the network capacity, by decreasing the mean access delay. Kwon et al. [6] proposed a mechanism that accelerates collision resolution and reduces idle slots.

This work proposes the Contention window Proactive Increase (CPI) to improve the IEEE 802.11 and the HomePlug standards. The CPI mechanism increases the throughput of both standards by reducing the number of collisions.

This work is organized as follows. Section 2 summarizes both IEEE 802.11 and HomePlug standards. Section 3 introduces the mechanism proposed and show its efficiency through simulations results. Section 4 concludes this work and investigates future directions.

\section{Access methods}

Both IEEE 802.11 and HomePlug use CSMA/CA (Carrier Sense Multiple Access with Collision Avoidance) to control the medium access. Collisions can not be detected due to attenuation and noise [7]. The sender can not assure whether a collision occurred at receiver.

Given the similarities of the electrical and wireless medium, their access methods have a lot of characteris- 
tics in common. Whenever a station wants to transmit a data frame, it must wait the medium remains idle for a defined interframe space plus a random time called backoff. As there is no guarantee of collision detection, the transmitter waits for an acknowledgment (ACK) from the receiver. The receiver transmits an ACK if it receives a data frame correctly. The ACK frame is transmitted after the end of the data frame reception plus another defined interframe space. The interframe spaces have different durations according to the standard.

The backoff procedure chooses a random number uniformly distributed between zero and the contention window size $[0, C W]$. This number is used as a backoff counter (BC) and its value times a slot time sets a backoff timer.

The stations decrement by one their BC if the medium remains idle for a slot time. They keep decrementing the counter until the backoff timer goes off and the transmission occurs. Nevertheless, if the medium is occupied while a station is waiting for backoff, the station must pause its backoff counter and wait for the next transmission attempt to resume it. The backoff procedure is called if the transmitter does not receive an ACK frame from the receiver. The station retransmits every time a data frame is transmitted and an ACK is not received.

The backoff procedure handles the increase of the contention window size. This size depends on the number of times the backoff procedure is called during the transmission of a specific frame. The backoff procedure counter (BPC) stores the number of calls to the backoff procedure. The backoff procedure is called whenever the ACK frame is not received and, as a consequence, a collision is assumed. Therefore, the contention window increase avoids collisions by decreasing their probability. In the first attempt to access the medium, a backoff counter between zero and the minimum $\mathrm{CW}$ size $\left[0, C W_{\text {min }}\right]$ is used. The increase in the $\mathrm{CW}$ size follows the expression $C W_{i}=2 \times C W_{i-1}+1$. This increase continues up to a maximum threshold $C W_{\max }$. If the backoff procedure is called when $C W=C W_{\max }$, the $\mathrm{CW}$ size is not changed. CW returns to its initial value whenever a successful transmission occurs.

A Network Allocation Vector (NAV) is set to keep the stations aware of the duration of the current transmission consequently avoiding collisions. The peculiarities of IEEE 802.11 and HomePlug are detailed in Sections 2.1 and 2.2, respectively.

\subsection{IEEE 802.11}

The IEEE 802.11 standard defines the MAC and physical layer specifications. In Ad Hoc mode, the stations that want to transmit must wait for DIFS (Distributed InterFrame Space), which lasts for $50 \mu \mathrm{s}$, plus a backoff interval. The slot time used by IEEE 802.11 is equal to $20 \mu$ s. Receiving the data frame correctly, the destination transmits the ACK frame after SIFS (Short Interframe Space), which lasts for $10 \mu \mathrm{s}$. The operation of IEEE 802.11 is depicted in Figure 1. Before the data transmission, the IEEE 802.11 allows an exchange of control frames, RTS/CTS (Request-ToSend/Clear-To-Send), to reserve the medium. The RTS/CTS mechanism is optional and will not be used in this work.

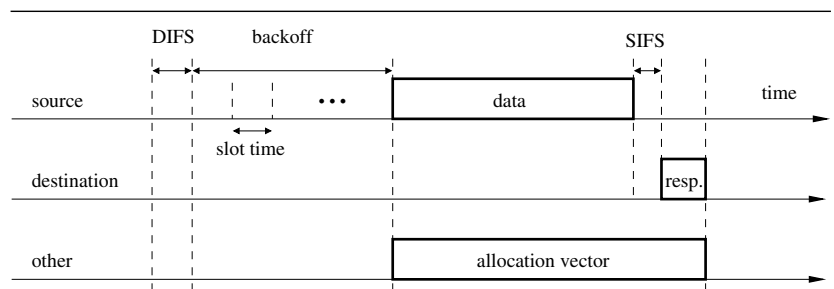

Figure 1. Transmission in IEEE 802.11.

The values of $\mathrm{CW}$ size according to the backoff procedure counter (BPC) are shown in Table 1.

\begin{tabular}{|c||c|c|c|c|c|c|}
\hline BPC & 0 & 1 & 2 & 3 & 4 & $\geq 5$ \\
\hline CW & 31 & 63 & 127 & 255 & 511 & 1023 \\
\hline
\end{tabular}

Table 1. IEEE 802.11 values for $\mathrm{CW}$.

\subsection{HomePlug}

In HomePlug 1.0, the medium must remain idle for $35.84 \mu$ s time interval called CIFS (Contention distributed InterFrame Space) before a frame can be transmitted. After CIFS, the stations get into the Priority Resolution (PR) phase. HomePlug supports to quality of service. Two slot times, PR0 and PR1, allow attempts to access the medium only from higher priority flows during backoff, as depicted in Figure 2. The intervals PR0 and PR1 also last for $35.84 \mu \mathrm{s}$ each. In this work, we use the minimum priority. After the PR phase, the station waits for the backoff time to transmit the data frame. The slot time used by HomePlug is equal to $35.84 \mu \mathrm{s}$. The destination sends an ACK after RIFS (Response InterFrame Space), which is equal to $26 \mu \mathrm{s}$.

The values of the CW size in HomePlug 1.0 and the corresponding values of BPC are shown in Table 2.

\begin{tabular}{|c||c|c|c|c|}
\hline BPC & 0 & 1 & 2 & $\geq 2$ \\
\hline CW & 7 & 15 & 31 & 63 \\
\hline DC & 0 & 1 & 3 & 15 \\
\hline
\end{tabular}

Table 2. HomePlug values for CW and DC.

HomePlug introduces the deferral counter (DC) mechanism conceived to avoid collisions by increasing the num- 


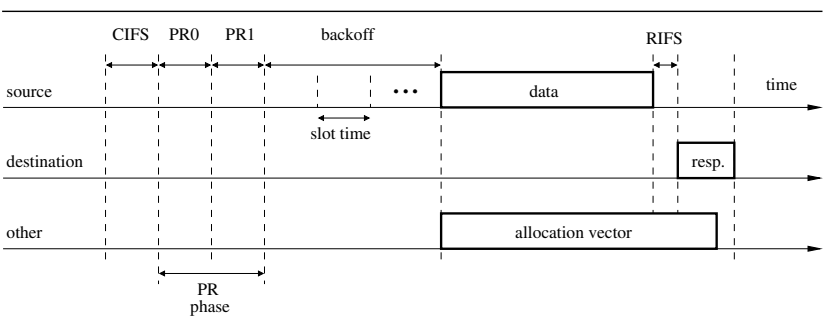

Figure 2. Transmission in HomePlug.

ber of times the backoff procedure is called during the transmission of one frame. Using DC, the backoff procedure may be called even if there was no collision. A station increases its BPC whenever a collision occurs or when DC reaches zero. During backoff, if another station transmits first, the station verifies its DC and decrements it if it is not zero. After decreasing DC, the station must pause its BC. The station resumes its $\mathrm{BC}$ and consequently its transmission if the medium gets idle for CIFS again and if its priority allows. If DC is zero, every station contending for the medium must perform a backoff procedure and postpone the transmission if it senses another ongoing transmission. After a backoff procedure, the stations will always use a new $\mathrm{CW}$ and $\mathrm{DC}$ values. This mechanism reduces the collision probability. If $\mathrm{CW}$ and DC reach the maximum values defined by the standard (Table 2), they are kept even if BPC is incremented. Receiving an ACK, the station resets the minimum values for $\mathrm{DC}$ and $\mathrm{CW}$.

\section{Contention window proactive increase}

This paper proposes the Contention window Proactive Increase (CPI) mechanism which is based on the HomePlug deferral counter (DC). The mechanism improves the collision avoidance in HomePlug and IEEE 802.11. The key idea is that the stations do not perform a backoff procedure only after a collision is assumed, but they also call the backoff procedure when the collision probability is high as in HomePlug. The CPI mechanism proposes a novel counter in HomePlug, similar to DC. Nevertheless, unlike HomePlug, the CPI mechanism uses a counter with fixed values independently of the backoff procedure counter (BPC). Using fixed values, the HomePlug reaction changes. Lower values implies in faster reaction. Additionally, we adapt the CPI mechanism to IEEE 802.11 motivated by the similarities of the two access methods. The CPI mechanism requires a small modification in firmware.

We propose the use of the CPI mechanism with a counter initialized with a constant. We tested different constants for HomePlug and IEEE 802.11. The results for the constants equal to 0,3 and 5 for HomePlug and equal to 0,3 and 10 for IEEE 802.11 are plotted. These values synthesize the be- havior of CPI.

\subsection{Results}

The efficiency of the CPI mechanism is verified through simulations using the ns-2 [3] simulator. We implemented the HomePlug module and modified the module available for IEEE 802.11. The simulation scenario is composed of nodes transmitting CBR over UDP traffic. The packet size is 1500 bytes and the number of stations varies from 1 to 16. All nodes are within the same transmission range and a null bit error rate is considered. The following results are computed with confidence intervals of $95 \%$ which are represented in the graphs by vertical error bars. The transmission rates used are the maximum allowed by IEEE $802.11 \mathrm{~b}$ and HomePlug 1.0. Thus, the IEEE 802.11 nodes transmit at $11 \mathrm{Mbps}$ and the HomePlug nodes at $14 \mathrm{Mbps}$.

Figures 3 and 4 evaluate the aggregated throughput achieved by IEEE 802.11 and HomePlug, respectively, with different values of the CPI counter. For IEEE 802.11 we use $k=0,3,10$ and for HomePlug, $k=0,3,5$.

Figure 3 shows that the CPI mechanism improves the throughput of the IEEE 802.11 standard for a high number of nodes. The efficiency of CPI increases when the collision probability is higher, because the contention window $(\mathrm{CW})$ is increased more frequently. Therefore, collisions are avoided and the throughput increases. Note also that the performance is better for small values of $k$ when the number of nodes is high. Smaller values of $k$ implies in a faster $\mathrm{CW}$ increase. The original IEEE 802.11 offers a higher throughput for a few number of nodes because the original standard defines a high value for $C W_{\min }$. Thus, initially the number of idle slots is high and the collision probability is low. A faster $\mathrm{CW}$ increase represents additional idle slots, reducing the throughput. The effect of the idle slots in IEEE 802.11 is observed in the throughput gain for a few number of nodes. Increasing the number of nodes from 1 to 2 , the contention for the medium reduces the average access time, increasing the throughput. This effect is also seen with $k=3$ and $k=10$.

Figure 4 shows that with $k=0$, the CPI improvement in HomePlug is independent of the number of nodes. Unlike IEEE 802.11, the $C W_{\min }$ value of HomePlug is already small and there are less idle slots. Thus, increasing the $\mathrm{CW}$ size, the collision probability decreases and the throughput gets higher. It is worth noting that using $k=3$ and 5 , the performance of CPI in HomePlug is worse than the original standard, because the reaction to congestions is slower. As a consequence, the number of collisions increases.

Additionally, we measured the average delay of each frame from the reception in the MAC sub-layer of the source, to the successful reception at the destination. The propagation delay is negligible. Figures 5 and 6 plot the av- 


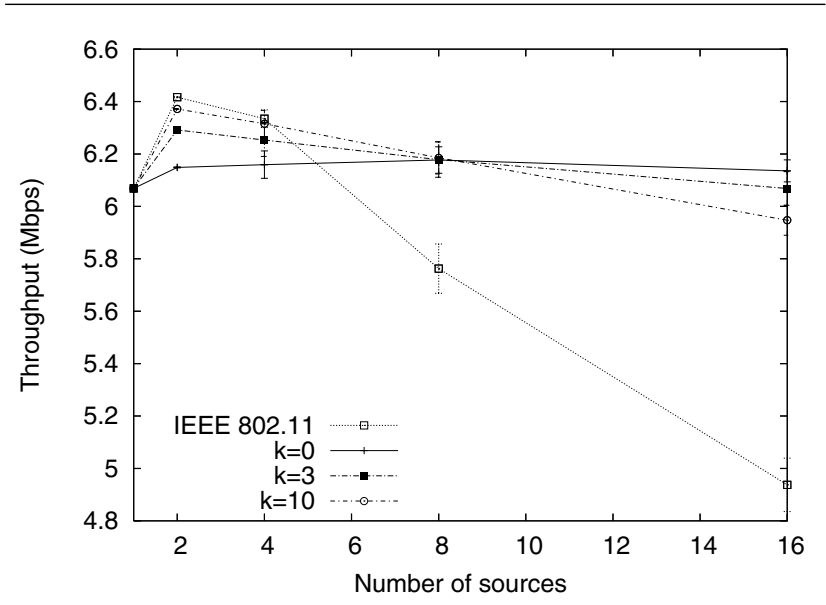

Figure 3. IEEE 802.11 throughput.

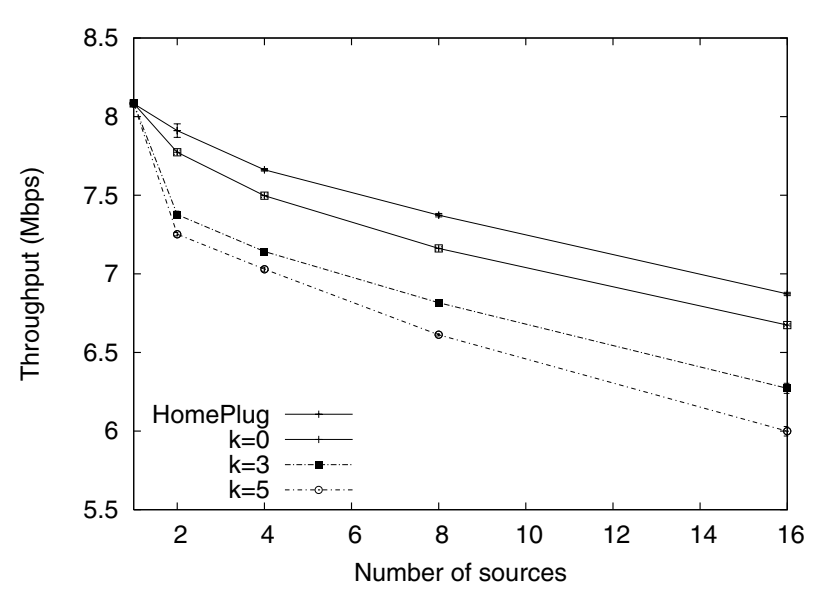

Figure 4. HomePlug throughput.

erage delay for IEEE 802.11 and HomePlug, respectively. Figure 5 shows that the delay of IEEE 802.11 is higher than the delay of the IEEE 802.11 with CPI. For a high number of nodes, the number of retransmissions increases with the number of collisions. As collisions are avoided more efficiently when using CPI, the delay is lower. Figure 6 shows that the delay of HomePlug with CPI is lower only for $k=0$. This is also due to the lower number of collisions. Using higher values of $k$, the CPI mechanism is not efficient and the average delay is higher.

A collision occurs whenever more than one station transmits in the same time slot. We define the collision percentage as the number of collisions divided by the total number of transmissions. Figures 7 and 8 show the collision percentage for IEEE 802.11 and HomePlug, respectively. Figure 7 shows that the collision percentage is higher for the original IEEE 802.11 for increasing number of nodes. This result agrees with the throughput results shown in Figure 3:

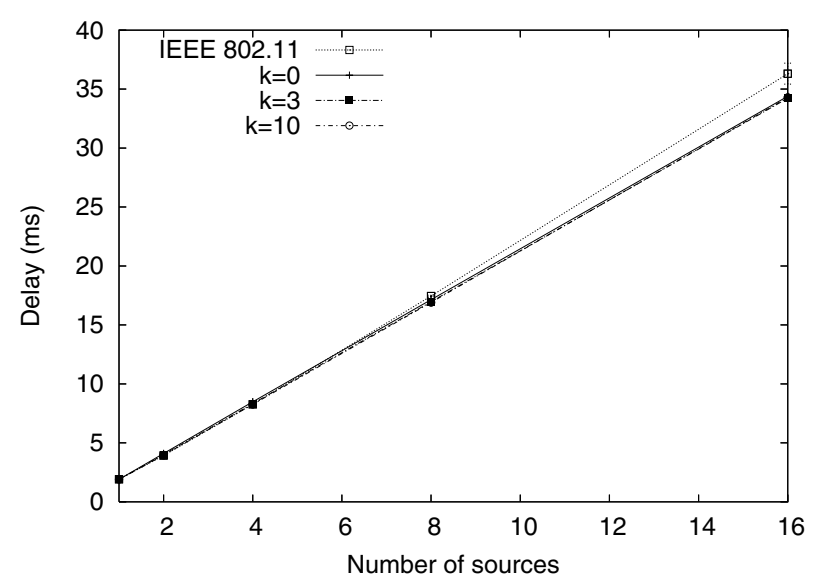

Figure 5. IEEE 802.11 average delay.

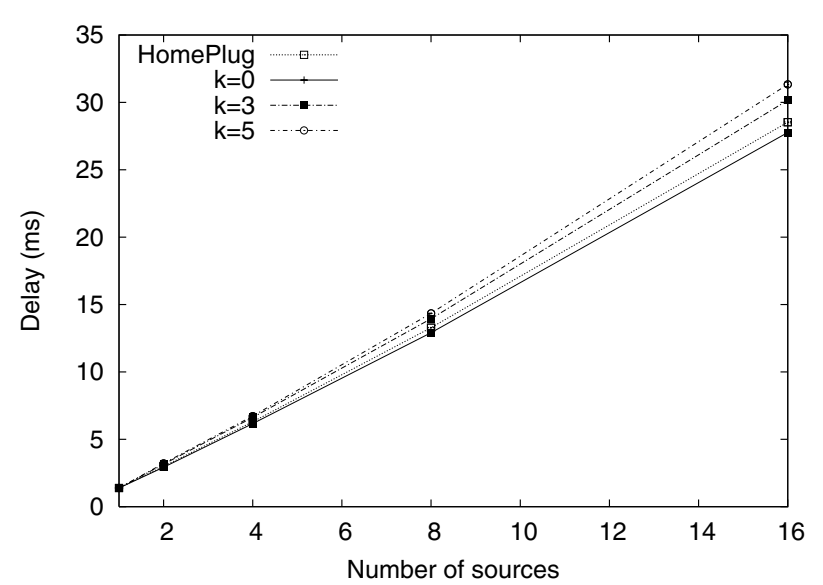

Figure 6. HomePlug average delay.

a lower number of collisions increases the throughput. Figure 8 shows that only with $k=0$ the collision percentage is lower than the original HomePlug, confirming the results in Figure 4 . The percentage of collisions is low for both standards when the number of nodes is small, because the number of nodes contending for the medium is small as well as the collision probability.

Finally, Figure 9 shows the efficiency of the CPI mechanism. We plot the throughput obtained using $k=0$ divided by the maximum rates of each standard, $14 \mathrm{Mbps}$ for HomePlug and 11 Mbps for IEEE 802.11. The CPI mechanism achieved a higher efficiency applied to IEEE 802.11 for a high number of nodes, because IEEE 802.11 does not use originally any DC-like mechanism. On the other hand, as HomePlug already uses DC, the efficiency gain of CPI is lower. For a smaller number of nodes, the efficiency of CPI is lower for IEEE 802.11 because of the idle slots which reduce the collision probability. In opposition, the efficiency 


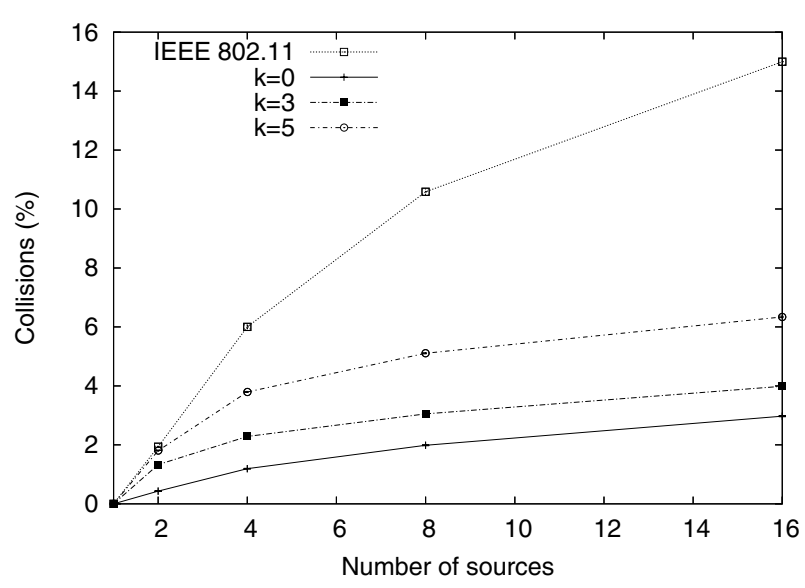

Figure 7. IEEE 802.11 collision percentage.

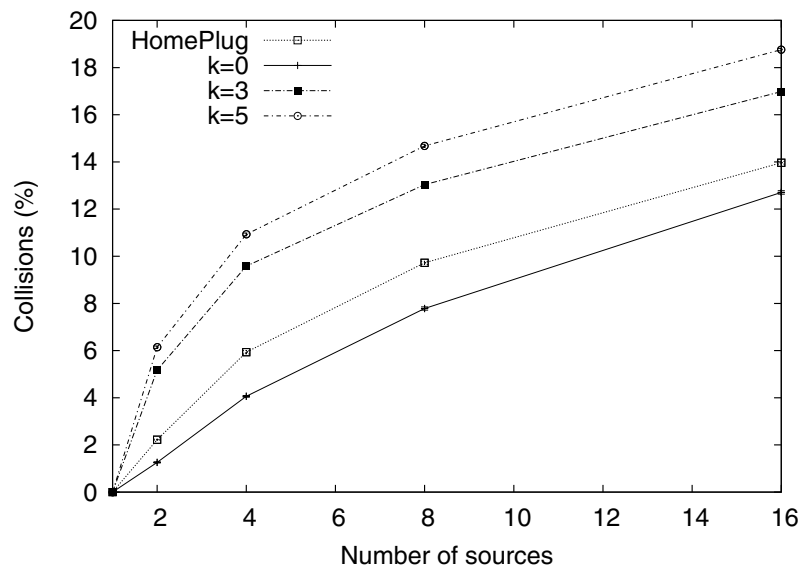

Figure 8. HomePlug collision percentage.

of CPI in HomePlug is higher because the collision probability is already considerable.

\section{Conclusions}

Wireless and PLC home networks are promising technologies due to characteristics like ubiquity and low-cost infrastructure. Nevertheless, challenges closely related to the transmission environment still exist.

In this paper, we proposed the Contention window Proactive Increase (CPI) mechanism to improve the throughput of the HomePlug and IEEE 802.11 MAC protocols. The CPI is a collision avoidance mechanism based on the HomePlug Deferral Counter (DC). Unlike the HomePlug DC, CPI defines a constant counter which is able to react faster against collisions. We verified the counter values which provide the best performance for each protocol. In IEEE 802.11, this value depends on the net-

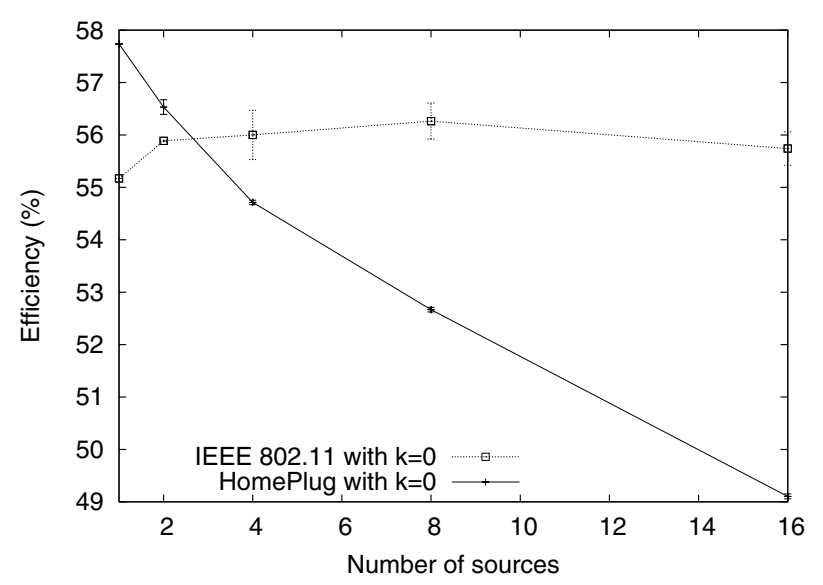

Figure 9. Efficiency.

work load and on the number of nodes. On the other hand, HomePlug showed a better performance when the backoff procedure is called whenever the deferring station senses another ongoing transmission, which is the effect of $k=0$.

Consequently, our future work will investigate the implementation of a dynamic mechanism which is able to adapt the values of the CPI counter to the network load.

\section{References}

[1] A. Amodei Jr., L. H. M. K. Costa, and O. C. M. B. Duarte. Increasing the Throughput of the HomePNA MAC Protocol. In IEEE LCN'2004, pages 294-301, Nov. 2004.

[2] L. Bononi, M. Conti, and L. Donatiello. Design and Performance Evaluation of a Distributed Contention Control (DCC) Mechanism for IEEE 802. 11 Wireless Local Area Networks. In Proc. ACM WoWMoM'98, pages 59-67, Oct. 1998.

[3] K. Fall and K. Varadhan. The ns Manual. UC Berkeley, LBL, USC/ISI, and Xerox PARC, Apr. 2005. Available at http://www.isi.edu/nsnam/ns/ns-documentation.html.

[4] IEEE. Wireless LAN medium access control (MAC) and physical layer (PHY) specifications. IEEE Standard 802.11, 1999.

[5] W.-K. Kuo and C.-C. Kuo. Enhanced Backoff Scheme in CSMA/CA for IEEE 802.11. In Proc. IEEE VTC-Fall, pages 2809-2813, Oct. 2003.

[6] Y. Kwon, Y. Fang, and H. Latchman. A Novel MAC Protocol with Fast Collision Resolution for Wireless LANs. In Proc. IEEE Infocom 2003, July 2003.

[7] M. K. Lee, R. E. Newman, H. A. Latchman, S. Katar, and L. Yonge. Homeplug 1.0 Powerline Communications LANs - Protocol Description and Performance Results. International Journal of Communication Systems, 16(5):447-473, June 2003.

[8] Y. C. Tay and K. C. Chua. A Capacity Analysis for the IEEE 802.11 MAC Protocol. Wireless Network, 7:159-171, Mar. 2001. 